\title{
The Worldwide Spread of the Tiger Mosquito as Revealed by Mitogenome Haplogroup Diversity
}

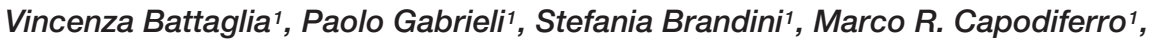 \\ Pio A. Javier ${ }^{2}$, Xiao-Guang Chen ${ }^{3}$, Alessandro Achilli ${ }^{1}$, Ornella Semino ${ }^{1}$, \\ Ludvik M. Gomulski ${ }^{1}$, Anna R. Malacrida ${ }^{1}$, Giuliano Gasperi ${ }^{1 *}$, Antonio Torroni ${ }^{*}$ and \\ Anna Olivieri ${ }^{1 *}$
}

\begin{abstract}
${ }^{1}$ Dipartimento di Biologia e Biotecnologie "L. Spallanzani", Università di Pavia, Pavia, Italy, ${ }^{2}$ Crop Protection Cluster, College of Agriculture, University of the Philippines Los Baños, Los Baños, Philippines, ${ }^{3}$ Department of Pathogen Biology, School of Public Health and Tropical Medicine, Southern Medical University, Guangzhou, China
\end{abstract}

\section{OPEN ACCESS}

Edited by:

Badri Padhukasahasram,

Illumina, USA

Reviewed by:

Gonzalo Gajardo,

University of Los Lagos, Chile

Octavio Salgueiro Paulo,

University of Lisbon, Portugal

Luisa Pereira,

Instituto de Patologia e Imunologia Molecular da Universidade do Porto,

Portugal

*Correspondence:

Anna Olivieri

anna.olivieri@unipv.it

Antonio Torron

antonio.torroni@unipv.it

Giuliano Gasperi

giuliano.gasperi@unipv.it

Specialty section:

This article was submitted to Evolutionary and Population Genetics, a section of the journal

Frontiers in Genetics

Received: 03 September 2016 Accepted: 09 November 2016

Published: 23 November 2016

Citation:

Battaglia V, Gabrieli P, Brandini S,

Capodiferro MR, Javier PA,

Chen X-G, Achilli A, Semino O,

Gomulski LM, Malacrida AR

Gasperi G, Torroni A and Olivieri A

(2016) The Worldwide Spread of the

Tiger Mosquito as Revealed by

Mitogenome Haplogroup Diversity.

Front. Genet. 7:208.

doi: 10.3389/fgene.2016.00208
In the last 40 years, the Asian tiger mosquito Aedes albopictus, indigenous to East Asia, has colonized every continent except Antarctica. Its spread is a major public health concern, given that this species is a competent vector for numerous arboviruses, including those causing dengue, chikungunya, West Nile, and the recently emerged Zika fever. To acquire more information on the ancestral source(s) of adventive populations and the overall diffusion process from its native range, we analyzed the mitogenome variation of 27 individuals from representative populations of Asia, the Americas, and Europe. Phylogenetic analyses revealed five haplogroups in Asia, but population surveys appear to indicate that only three of these (A1a1, $A 1 a 2$, and $A 1 b)$ were involved in the recent worldwide spread. We also found out that a derived lineage (A1a1a1) within A1a1, which is now common in Italy, most likely arose in North America from an ancestral Japanese source. These different genetic sources now coexist in many of the recently colonized areas, thus probably creating novel genomic combinations which might be one of the causes of the apparently growing ability of $A$. albopictus to expand its geographical range.

Keywords: Aedes albopictus, tiger mosquito, mitochondrial DNA, mitogenomes, haplogroups

\section{INTRODUCTION}

The genus Aedes includes five highly invasive species, A. albopictus, A. aegypti, A. j. japonicus, A. koreicus, and A. atropalpus. Of these, A. albopictus and A. j. japonicus are the most widespread across the globe, and A. aegypti and A. albopictus, being competent vectors for several human tropical diseases, have a major impact on human health. The North American species, A. atropalpus, arrived in Europe (Italy, France, and the Netherlands) through international trade (Romi et al., 1997), but it was subsequently exterminated in Italy and France and is unlikely to have established in the Netherlands due to climatic conditions (Scholte et al., 2009). Aedes j. japonicus and A. koreicus, native to East Asia (Japan, Korea, China, Russia), have both colonized central Europe and A. j. japonicus is also widely distributed in the US (Medlock et al., 2015). Aedes aegypti originated in sub-Saharan Africa and is now considered the main vector of dengue. Climate appears to be the decisive factor limiting the distribution of $A$. aegypti to tropical and sub-tropical regions, with few incursions into Europe and North America (Powell and Tabachnick, 2013; Khormi and Kumar, 2014). 
In contrast, $A$. albopictus, indigenous to East Asia, is not so restrained by climatic factors, and in the last 40 years has successfully colonized the tropical and temperate regions of all continents (Benedict et al., 2007; Paupy et al., 2009; Bonizzoni et al., 2013; Kraemer et al., 2015). This mosquito has become a growing public health concern, being a competent vector for many arboviruses which cause lethal or debilitating human diseases, including the dengue (DEN), chikungunya (CHIK), West Nile (WN) viruses (Gasperi et al., 2012; Bonizzoni et al., 2013), and the recently emerged ZIKA virus (Wong et al., 2013; Chouin-Carneiro et al., 2016). Although it has been considered a less efficient vector than $A$. aegypti, this species is the sole vector of recent DENV outbreaks in Southern China, Hawaii, the Indian Ocean and Gabon and the first autochthonous DENV transmission in France and Croatia (Paupy et al., 2009; Wu et al., 2010; Peng et al., 2012; Rezza, 2012, 2014; Schaffner et al., 2013). A. albopictus was most likely the main DENV vector in Asia prior to the introduction of $A$. aegypti in the mid nineteenth century (Gubler, 2006).

Introduced into Europe (Albania) in 1979, A. albopictus has now colonized all Mediterranean countries from Spain to Syria and has been reported in Central Europe (Medlock et al., 2012). It was introduced into Hawaii at the end of the 19th century (Rai, 1991) and into continental USA (Texas) in 1985, and is now well established in 32 states. Its presence has been reported in Mexico (first recorded in 1988), Central and South America (first recorded in Brazil, 1986), and Africa (first recorded in South Africa, 1989; Bonizzoni et al., 2013). Its ability to spread from the native range and adapt to local environments is probably due to its ecological characteristics, drought-resistant eggs with the ability to diapause, daylight biting habit, aggressive and opportunistic feeding behavior, and capacity to achieve high population densities (Paupy et al., 2009).

The nuclear genome of the tiger mosquito from two laboratory strains, the Italian Fellini (an isofemale line derived from the Rimini strain; Bellini et al., 2007; Dritsou et al., 2015) and the Chinese Foshan strain (Chen et al., 2015), was recently published, but only two complete mitogenomes $(\sim 16.7 \mathrm{~kb})$ are available in GenBank, from Taiwan (Asian tiger mosquito Reference Sequence, NC006817) and from Nanjing, Jiangsu Province, China (KR068634; Zhang et al., 2015). Despite the availability of mitogenomes, virtually all A. albopictus mitochondrial DNA (mtDNA) surveys were restricted to short segments of the cytochrome $\mathrm{c}$ oxidase subunit 1 (COI) and/or NADH dehydrogenase subunit 5 (ND5) genes, suggesting a limited phylogeographic differentiation among populations, possibly also caused by the inclusion in these studies of laboratory stocks or sibling eggs (Delatte et al., 2011; Kamgang et al., 2011, 2013; Porretta et al., 2012; Zhong et al., 2013; Zawani et al., 2014; Futami et al., 2015) and the postulated cytoplasmatic sweep caused by Wolbachia infection (Armbruster et al., 2003). However, more extensive sequencing of the COI gene has revealed more variation than previously thought (Goubert et al., 2016), a scenario also partially supported by microsatellite studies that highlighted slight genetic diversity between native and adventive populations with high variability within populations (Manni et al., 2015).

Previous studies have shown that the variation seen in short mtDNA segments may be inadequate to both identify and phylogenetically link haplogroups (Torroni et al., 2006; Achilli et al., 2008, 2012). This is especially true for the insect mtDNA control region due to its peculiar features: high $\mathrm{A}+\mathrm{T}$ content and reduced substitution rate, variable size and high length mutation rate, concerted evolution of tandem repeats and directional mutation pressure (Zhang and Hewitt, 1997).

To identify the ancestral source(s) of $A$. albopictus adventive populations, overcoming previous limitations, we here determined and analyzed the sequence variation at the level of entire coding regions of 27 mitogenomes ( 25 novel and two previously published) from Eastern and Southeastern Asian, American, and European populations. Our analyses reveal that only three of the five identified Asian haplogroups, which are differentially distributed in Asian populations living in temperate and tropical regions, were involved in the recent worldwide spread. These different ancestral sources from Asia now coexist in many adventive populations with possible implications for the adaptive capability of the species.

\section{MATERIALS AND METHODS}

\section{Sample Collection and DNA Extraction}

A total of 25 novel mitogenomes were included in this study. Twenty-two were from wild populations collected in Europe, Asia, and the Americas (Table 1; Figure 1). Three were from the Americas (two from Virginia and one from Brazil). Nine were from Asia: three from Thailand (one from Hang Chat district, Lampang province in the North; one from Ban Rai district, Uthai Thani province in the West; one from Phato district, Chumphon province in the South), five from Los Baños, Laguna, Philippines and one from Wakayama prefecture, Japan. Ten were from Europe: two from Tirana (Albania), two from Athens (Greece), two from Cesena and two from Pavia (Northern Italy), one from Cassino (Central Italy), and one from Reggio Calabria (Southern Italy). This study also included three adult laboratory-maintained strain mosquitoes: two from the Italian Rimini strain (Bellini et al., 2007; Manni et al., 2015), established at CAA (Centro Agricoltura Ambiente "G. Nicoli," Crevalcore, Italy) from mosquitoes collected in Rimini, Italy, and one from the Chinese Foshan strain (Center for Disease Control and Prevention of Guangdong Province; Table 1). The study did not involve protected species and specimens were not collected at sites protected by law.

Morphological keys (Rueda, 2004) and/or PCRs with speciesspecific primers for internal transcribed spacer regions (ITS1 and ITS2) of ribosomal DNA (rRNA; Higa et al., 2010) were used to identify the specimens. For the Philippine samples, eggs were collected using ovitraps, and the emerging adults were reared in an insectary under standard conditions of temperature $\left(27^{\circ} \mathrm{C}\right)$, humidity $(60-80 \%)$, and photoperiod (12:12 h). Samples were preserved in $80 \%$ ethanol and stored at $-20^{\circ} \mathrm{C}$ until 
TABLE 1 | Origin and haplogroup affiliation of $A$. albopictus mitogenomes considered in this study.

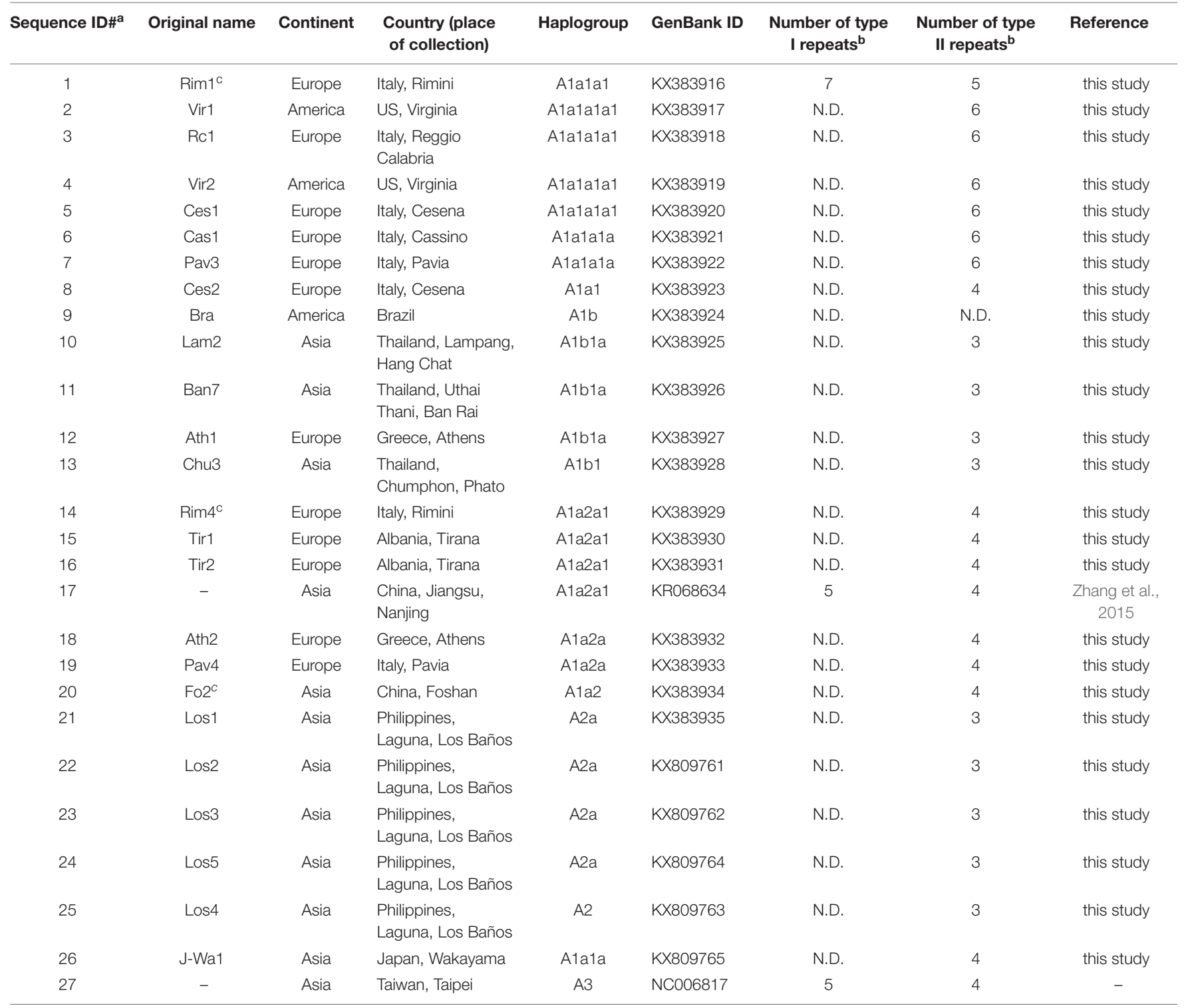

a/D numbers correspond to those in Figure 1.

${ }^{\mathrm{b}} \mathrm{N} . \mathrm{D} .=$ not determined.

${ }^{\mathrm{C}}$ Laboratory-maintained strain.

DNA extraction. DNA extraction was carried out using the Wizard Genomic DNA Purification Kit (Promega) following the manufacturer's protocol.

\section{Sequencing of the $A$. albopictus Mitochondrial Coding and Control Regions}

A primer set was designed to amplify the entire A. albopictus mitogenome in four overlapping PCR fragments (protocol I). The A. albopictus Reference Sequence (NC006817) was used to design primer sets. The coding region (nps 1-14893) was amplified by two long PCR fragments whereas the control region (nps 14894-16665) was amplified by two rather short PCR fragments
(Supplementary Table 1) because of its high $\mathrm{A}+\mathrm{T}$ content and the presence of repeated elements requiring distinctive PCR procedures.

Coding region long PCRs (Supplementary Table 1) were carried out in $50 \mu \mathrm{l}$ reaction mixture containing $1 \mathrm{X}$ GoTaq Long PCR Master Mix (Promega), $0.2 \mu \mathrm{M}$ of each primer and 10$20 \mathrm{ng}$ of DNA template using the following PCR conditions: $94^{\circ} \mathrm{C}$ ( $2 \mathrm{~min}) ; 30$ cycles of $94^{\circ} \mathrm{C}(30 \mathrm{~s}), 59^{\circ} \mathrm{C}(30 \mathrm{~s}), 65^{\circ} \mathrm{C}(9 \mathrm{~min})$; and a final extension of $72^{\circ} \mathrm{C}(10 \mathrm{~min})$. Alternatively, a set of nine overlapping PCR fragments (Supplementary Table 2) covering the A. albopictus coding region was also designed (protocol II). PCRs were carried out in $25 \mu \mathrm{l}$ reaction with a standard reaction mix containing $1 \mathrm{X}$ White Buffer $\left(1.5 \mathrm{mM} \mathrm{MgCl}_{2}\right), 0.2 \mathrm{mM}$ of each dNTP mix, $0.6 \mathrm{U}$ of GoTaq G2 Polymerase (Promega), $0.2 \mu \mathrm{M}$ of 


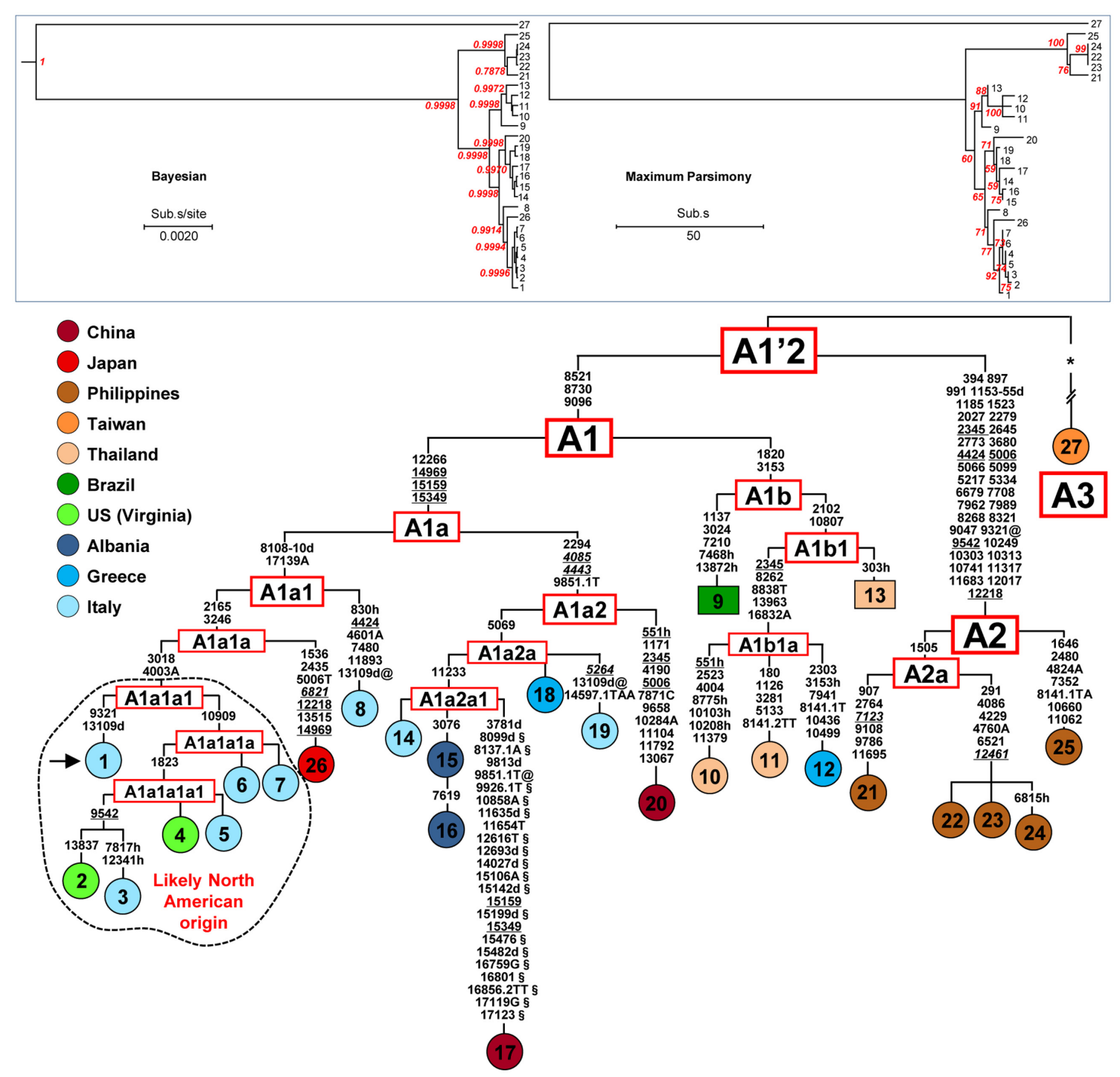

FIGURE 1 | Phylogeny of $\boldsymbol{A}$. albopictus mitogenomes. The Bayesian (left) and MP (right) trees are shown in the top inset. The posterior probability for the major nodes in the Bayesian tree is reported in red, whereas bootstrap values (1000 replications) are reported in red in the MP tree. These trees encompass 25 novel and two previously published sequences (Table 1). A magnified MP tree is also shown reporting all mutations that characterize the 27 mitogenomes except those linking mitogenome \#27 to the A1'2 node (see below). The asterisk (*) indicates the location of these mutations (347 in the coding region and 16 in control region), which are listed in Supplementary information for Figure 1. For the phylogeny construction, the entire coding region variation of all mitogenomes was included as well as some control region mutations (see "Material and Methods"). The published sequences (\#17 and \#27, Table 1) are from the Jiangsu Province, China (Zhang et al., 2015) and from Taipei, Taiwan, respectively. The mitogenome from a mosquito of the Italian Rimini strain (\#1, marked by the arrow) was employed to number the mutations shown on the branches. Mutations are transitions unless a base is explicitly indicated for transversions (to $A, G, C$, or $T)$ or a suffix for indels $(0.1, d)$. Heteroplasmic positions are marked by an "h." Recurrent mutations within the phylogeny are underlined (and in italics if present in mitogenome \#27) and back mutations are marked with the suffix @. The numerous mutations shared only by the published mitogenomes \#17 and \#27 are marked with the suffix §. Taking also into account that, despite their extensive coding region differentiation, mitogenomes \#17 and \#27 intriguingly harbor virtually identical control region sequences, it is likely that at least some of the mutations marked with the suffix $\S$ are mistakes. Colors illustrate geographic origins. Table 1 provides additional information concerning the geographic origin and haplogroup affiliation of each sample. Length variation (insertions/deletions) in a poly-A stretch beginning at $\mathrm{np} 3808$ was not considered. Note that for mitogenomes \#9 and \#13 (in squared boxes) the sequence variation was assessed only partially for the coding region and not at all for the control region (Supplementary information for Figure 1). The sub-haplogroup A1a1a1 and its derivatives of probable North American origin are encircled.

each primer and 20-30 ng of DNA template, using the following PCR conditions: $94^{\circ} \mathrm{C}(2 \mathrm{~min}) ; 35$ cycles of $94^{\circ} \mathrm{C}(30 \mathrm{~s}), 55^{\circ} \mathrm{C}$ (30 s), $72^{\circ} \mathrm{C}(2 \mathrm{~min})$; and a final extension of $72^{\circ} \mathrm{C}(5 \mathrm{~min})$.

PCR primers used to amplify the control region in two overlapping fragments (Supplementary Table 1) were the same in both protocols. The control region PCRs were carried out using the following PCR conditions: $94^{\circ} \mathrm{C}(2 \mathrm{~min}) ; 35$ cycles of $94^{\circ} \mathrm{C}$ $(30 \mathrm{~s}), 54^{\circ} \mathrm{C}(30 \mathrm{~s}), 60^{\circ} \mathrm{C}(2 \mathrm{~min})$; and a final extension of $60^{\circ} \mathrm{C}$ (5 min) for PCR \#3, and $94^{\circ} \mathrm{C}(2 \mathrm{~min}) ; 40$ cycles of $94^{\circ} \mathrm{C}(30 \mathrm{~s})$, $55^{\circ} \mathrm{C}(30 \mathrm{~s}), 60^{\circ} \mathrm{C}(1 \mathrm{~min})$; and a final extension of $60^{\circ} \mathrm{C}(10 \mathrm{~min})$ 
for PCR \#4. PCR products were visualized on a $1-2 \%$ agarose gel and successful amplicons were sequenced with standard dideoxy sequencing using Big Dye v3.1 Chemistry (Applied Biosystems) on 3730xl and 3130xl Genetic Analyzer (Applied Biosystems) following the manufacturer's protocol. Sets of 28 and 29 oligonucleotides were designed to sequence the A. albopictus mtDNA coding region starting from protocol I (Supplementary Table 3) or protocol II (Supplementary Table 4), respectively. Sequences were assembled and aligned using Sequencher 5.0 (Gene Codes) comparing them with the Reference Sequence (NC006817) from a Taipei sample, Taiwan.

\section{Cloning and Sequencing of Mitogenome \#1 (Rimini Strain) Control Region}

Given the different copy numbers of repeated elements contained in the A. albopictus control region, PCR fragments \#3 and \#4 (Supplementary Table 1) yielded products of different length in different mosquitoes. The two types of tandem repeats, I and II (Figure 2), were amplified with PCR \#3 and PCR \#4, respectively. Overall, the size of the amplified fragments ranged from $\sim 1,800$ to $\sim 2,500 \mathrm{bp}$ for PCR \#3 and from $\sim 800$ to $\sim 900 \mathrm{bp}$ for PCR \#4. As for one of the two mitogenomes from the Rimini strain (mitogenome \#1, Figure 1; Table 1), PCR \#3 and \#4 yielded products of $\sim 2,000 \mathrm{bp}$ and $\sim 900 \mathrm{bp}$, respectively. The product of PCR \#4 was directly cloned in the pCR2.1 TOPO vector (Invitrogen) following the manufacturer's protocol. White colonies were PCR-screened for the insert length and desired clones were sequenced bi-directionally using the M13 universal primers. The procedure described above is inefficient for longer fragments such as that of $\sim 2,000 \mathrm{bp}$, therefore a different strategy was developed to sequence the region amplified by PCR \#3. It was re-amplified in two overlapping PCR fragments (PCRs I and II), each with one primer within the tandemly repeated elements of type I (Supplementary Table 5). Multiple amplicons were obtained for each PCR and were cloned as described above. Only the two clones containing the longest fragments deriving from each PCR were sequenced bi-directionally. Sequences for each clone were assembled and aligned using Sequencher 5.0 (Gene Codes). Two additional internal primers were then newly designed (data not shown) to confirm the sequence obtained by cloning.

\section{Phylogeny Construction}

The obtained complete sequence of mitogenome \#1 (accession number KX383916) was used to assemble and number, with Sequencher 5.0 (Gene Codes), the other 24 novel mitogenomes (accession numbers KX383917-35, KX809761-65). We aligned the novel sequences and the two previously published mitogenomes - \#17 from China (Zhang et al., 2015) and \#27 from Taiwan (NC006817) - by performing a Multiple-Sequence Alignment with the Clustal algorithm (Chenna et al., 2003) implemented by Sequencher 5.0.

Most Parsimonious (MP) trees (1000 bootstrap replications) encompassing the 27 mitogenomes were built by using MEGA7 (Kumar et al., 2016), employing the Tree-Bisection-Regrafting (TBR) algorithm (Nei and Kumar, 2000). Modelgenerator v.85 indicated for our dataset $\mathrm{HKY}+\mathrm{I}$ as the best-supported model according to the AIC1, AIC2, and BIC criteria. The obtained settings were selected to infer maximum likelihood (ML) and Bayesian trees for the A. albopictus dataset. The ML tree was built using PAMLX (Yang, 2007) and assuming the HKY85 mutation model (two parameters in the model of DNA evolution) with gamma-distributed rates (approximated by a discrete distribution with eight categories). The Bayesian tree was obtained using BEAST 1.8.3 (Drummond and Rambaut, 2007) and running 50,000,000 iterations, with samples drawn every 10,000 Markov chain Monte Carlo (MCMC) steps. It was visualized using FigTree v.1.4.2. Phylogeny reconstruction was performed considering all the nucleotide substitutions (excluding indels and heteroplasmies) in the coding region (from np 1 to np 14896, relative to mitogenome \#1) and the five informative control region mutations 14969, 15159, 15349, 16832A, and 17139A. Thirteen additional control region mutations (see Supplementary information for Figure 1), ten shared exclusively by the previously published mitogenomes \#17 and \#27 and whose reliability is doubtful, and three (private mutations) seen only in mitogenome \#27, were simply superimposed on the magnified MP tree (Figure 1).

\section{Survey of Published Partial COI and ND5 mtDNA Sequences}

The identification of haplogroup diagnostic mutations allowed us to survey $1170 \mathrm{~A}$. albopictus partial COI and ND5 available in the literature for the presence/absence of these mutations. Of these, 284 mtDNAs are from Asia, 349 from the Americas, 32 from Europe, 153 from Africa, and 352 from Oceania (Table 2), thus encompassing populations from both native and non-native areas, and living at different climatic conditions.

\section{RESULTS}

\section{The Variation of $A$. albopictus mtDNA Control Region}

The first step in our study was to sequence the entire mtDNA (mitogenome \#1) from one mosquito of the Italian (Rimini) laboratory-maintained strain (Table 1; Figure 1), whose nuclear genome was recently sequenced (Dritsou et al., 2015). This mitogenome sequence confirmed that the A. albopictus control region belongs to group 2 of insect control regions (Zhang and Hewitt, 1997), like that of A. aegypti (Dueñas et al., 2006). Three conserved blocks are positioned along the region, which contains two different types (I and II) of tandem repeats (Figure 2). Type I consists of a $\sim 190$ bp repeat unit, whereas type II is made up of a short unit of $\sim 42 \mathrm{bp}$. The number of type I and type II tandem repeats that we observed in mitogenome \# 1 was different from those in sequence NC006817 from Taiwan (mitogenome \#27 in Figures 1 and 2) and variable among mtDNAs (Table 1 and data not shown). Moreover, between these two types of repeats, delimited by two conserved blocks, lies an $\mathrm{A}+\mathrm{T}$ rich region of variable length. The overall length and tandem repeat composition make the PCR amplification and sequencing of 


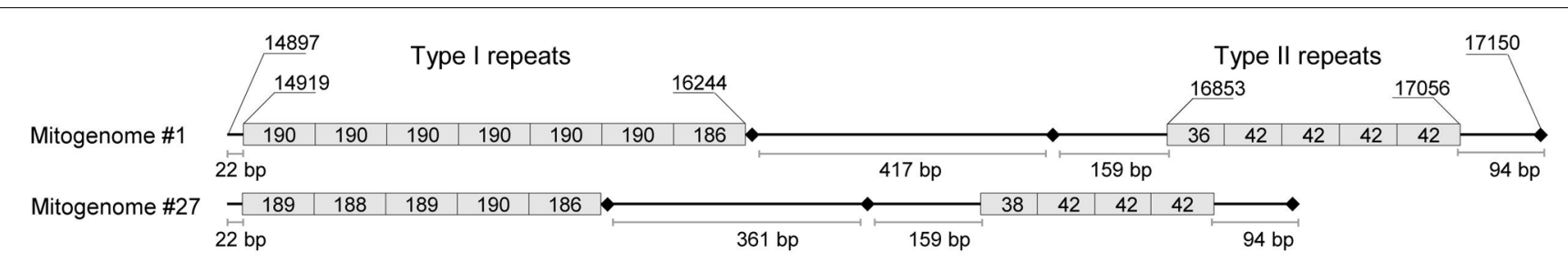

FIGURE 2 | Organization of tandem repeats in the control region of $\boldsymbol{A}$. albopictus mitogenomes. This schematic representation is based on the complete sequence information of mitogenomes \#1 (Rimini strain) and \#27 (Figure 1; Table 1). Two types of repeats (I and II) were observed with copy number differing for both repeats in the two mitogenomes. Type I consists of a $\sim 190$ bp repeat unit whereas type II is made up of a shorter unit of $\sim 42$ bp. The exact length (in bp) of each repeat is reported inside the box. The size of boxes representing repeats is not proportional to repeat lengths. Shown nucleotide positions are relative to sequence \#1. Variation in size due to variable number of tandem repeats in the two regions, when available, is provided in Table 1. Black diamonds represent in the order from left to right: a poly-T stretch motif, a poly-T stretch motif followed by a GC-rich block, and a short poly-A stretch motif. All of these are conserved in different mosquito species (Dueñas et al., 2006).

the entire $A$. albopictus control region extremely difficult (see "Material and Methods"). For the reasons outlined above, and difficulties originating from the impossibility of distinguishing heteroplasmy from PCR artifacts (due to replication slippage), we restricted our sequencing of A. albopictus mitogenomes to the coding region (from np 1 to $\mathrm{np}$ 14893, NC006817) and nearby control region segments. This approach was employed to obtain the coding region sequences of the additional 24 mitogenomes.

\section{MtDNA Haplogroups in A. albopictus}

Figure 1 illustrates the Bayesian and MP trees derived from the coding regions of the $27 \mathrm{~A}$. albopictus mitogenomes (25 novel and two previously published). The overall tree structure is virtually identical with the two approaches and is supported by the ML tree (Supplementary Figure 1), indicating a high degree of internal consistency for all major branches. A magnified MP tree is shown in the lower part of Figure $\mathbf{1}$ in order to illustrate the branch location of the identified mutations.

The mitogenomes (24 distinct haplotypes) cluster into three major branches that we named haplogroups A1, A2, and A3. Haplogroup A1 includes 21 mitogenomes, A2 consists of the five Philippine samples, while A3 encompasses only one mitogenome (\#27) from Taiwan. Haplogroups A1 and A2 are rather close to each other and to the $\mathrm{Al}^{\prime} 2$ node. In contrast, the $\mathrm{A} 3$ mitogenome differs by 363 mutations from the same node.

Overall, this phylogeny reveals an extensive and previously unreported mitogenome differentiation within A. albopictus. Indeed, when calculated on the standard COI sequence (Hebert et al., 2003) employed for DNA barcoding, the maximum intraspecific divergence was 0.012 (eight mutations in $658 \mathrm{bp}$ ), a value in line with those recently reported for $A$. scutellaris (0.008) and $A$. aegypti (0.022), but much greater than the value previously reported for $A$. albopictus (0.002; Sumruayphol et al., 2016).

Haplogroup A1, which encompasses most of the mitogenomes in the phylogeny, is subdivided into two branches that we termed A1a and A1b, with the former further split into A1a1 and A1a2. In our phylogeny these clades and subclades appear to be correlated with different geographic distributions. The branch Alal includes the single mitogenome from Japan, the two mitogenomes from the US (Virginia) and many of the mitogenomes from Italy, including one (\#1) of the two detected in the Rimini laboratory strain. The mitogenome from Japan (\#26) departs from the node A1ala and its sister clade Alala1 contains a sub-branch, Alalala1, of particular interest. It consists of four mitogenomes, two from Italy and the two from the US mentioned above. One of the US mitogenomes (\#4) is identical to one from Northern Italy (\#5) while the second $(\# 2)$ is closely related to the mitogenome \#3 from Southern Italy.

The sister branch A1a2 is formed by mitogenomes from different regions of Southern Europe (Italy, Albania, and Greece), including the second mitogenome (\#14) from the Rimini strain, the previously published Chinese sequence [\#17, KR068634 (Zhang et al., 2015)] and the mitogenome (\#20) from the Chinese Foshan strain, a laboratory-maintained colony founded in 1981 from mosquitoes from Southeast China. It is worth mentioning that the presence of two distinct haplotypes in different subjects of the Rimini laboratory-maintained strain, one belonging to A1a1a1 and the second to A1a2a1, reveals that at least two females contributed to the genetic formation of the strain. Haplogroup Alb contains all the mitogenomes from Thailand, which cluster in its A1b1 sub-branch, as well as one from Greece and one from Brazil. Finally, haplogroup A2 consists of multiple haplotypes, all from the Philippines.

\section{Haplogroup Affiliation of Worldwide mtDNA Sequences from $A$. albopictus}

The phylogenetic analysis not only allowed the identification of $A$. albopictus haplogroups and sub-haplogroups but also the definition of their distinguishing mutations (Figure 1). These include some diagnostic markers that are located in COI and ND5 partial sequences whose variation has been extensively assessed by published studies and can be retrieved from GenBank (Table 2).

By surveying these sequences for the presence or absence of these mutations, we were able to determine the most likely haplogroup affiliation for most of the 1170 tiger mosquito mtDNAs from populations worldwide. Table 2 reports the 
TABLE 2 | Frequencies of $A$. albopictus mtDNA haplogroups in worldwide populations.

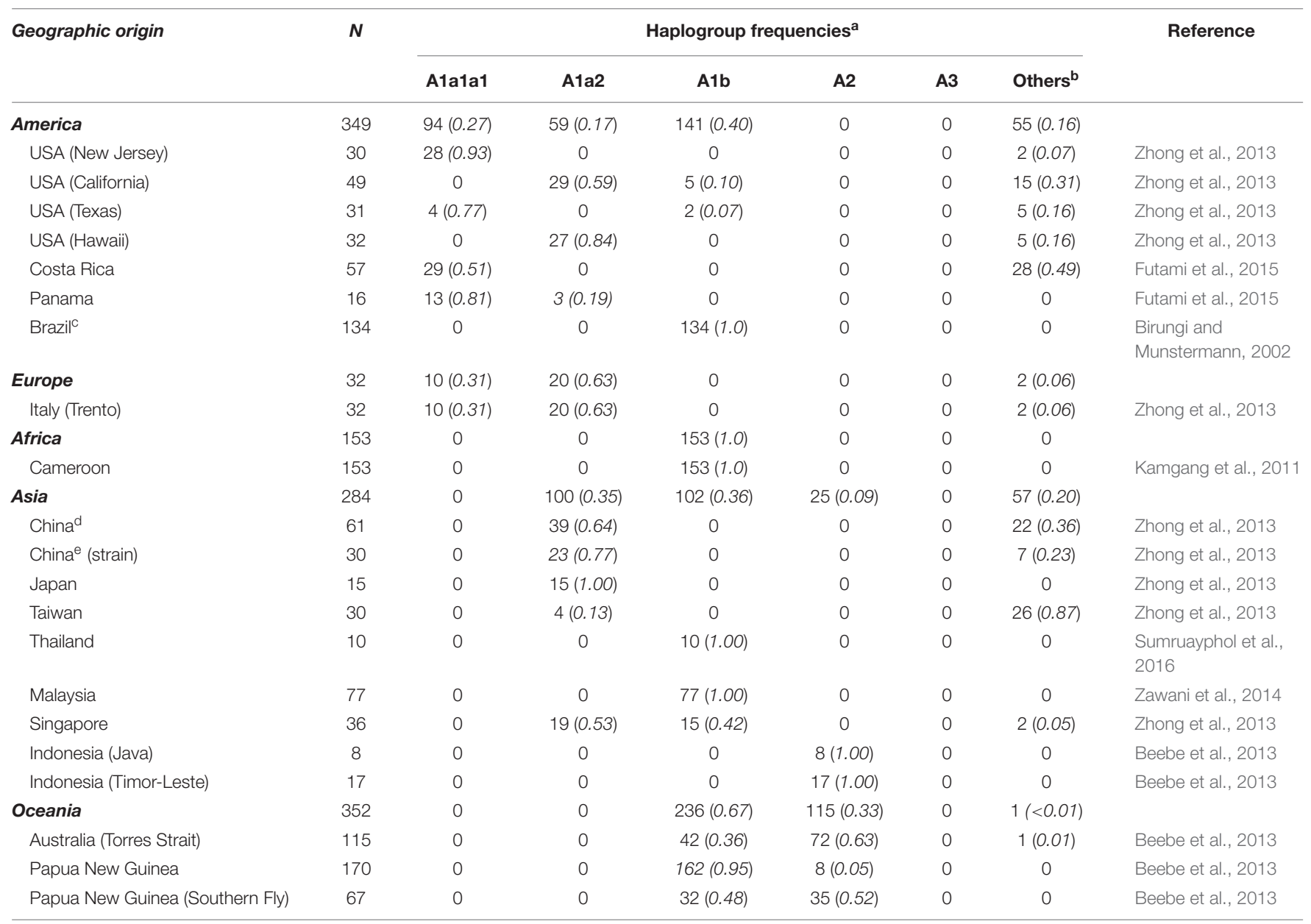

a Haplogroup affiliation is based on the sequence variation of the cytochrome c oxidase subunit I (COI) gene, except for the Brazilian samples (see footnote c). The diagnostic mutations are the lack of the transitions at nps 2165 and 1536 for haplogroup A1a1a1, the presence of the transition at np 2294 for haplogroup A1a2, the presence of the transition at $\mathrm{np} 1820$ for haplogroup A1b, and the presence of the transition at np 2027 for haplogroup A2.

'These samples were classified as "others" because they harbor the transition at np 2165 (except those from Australia, in which that nucleotide position was not sequenced), thus they do not belong to haplogroup A1a1a. Moreover, they lack the transitions at nps 2294, 1820, and 2027, thus they are not members of haplogroups A1a2, A1b, nor A2. Some additional mutations found in these samples are shared between specimens from the same or different geographic areas. In most cases they are likely due to multiple mutational events (recurrent mutations), but some might instead mark additional and not yet identified haplogroups.

'Samples from Brazil were classified on the basis of their NADH dehydrogenase subunit 5 (ND5) gene sequences. They were considered members of haplogroup A1b because they harbored the transition at np 7210.

${ }^{\mathrm{d}}$ Samples are from Guangdong and Fujian provinces.

e Laboratory-maintained strain, Jiangsu province.

haplogroup frequencies for A1a1a1, A1a2, A1b, A2, and A3 obtained from this survey, as well as a category termed "others" that includes mtDNAs that we could not classify and might encompass haplogroups not represented in our phylogeny. Figure 3 provides an overview of the worldwide spatial distribution of these haplogroups.

\section{DISCUSSION}

\section{Geographical Distribution of A. albopictus mtDNA Haplogroups}

Phylogenetic analyses revealed that our A. albopictus mitogenomes cluster into the three main haplogroups A1,
$\mathrm{A} 2$, and A3. Intriguingly, the population screening of the COI mutations $(1503,1578 \mathrm{C}, 1676 \mathrm{C}, 1704,1964)$ found in mitogenome \#27 from Taiwan, the only one belonging to haplogroup A3 in our phylogeny, did not reveal any match in the 1170 tiger mosquito mtDNAs from worldwide populations, not even in the population sample $(N=30)$ from Taiwan (Table 2). This suggests that $A$. albopictus mosquitoes with $\mathrm{A} 3 \mathrm{mtDNAs}$ were probably not involved in the recent worldwide spread of the species, raising the possibility that this haplogroup might be rare and/or with a restricted geographical distribution.

In contrast, the survey for COI mutations characterizing the Philippine mitogenomes \#21-25 allowed the identification of many other mtDNAs belonging to haplogroup A2 (Table 2), but all in Insular Southeast Asia, suggesting that this haplogroup 


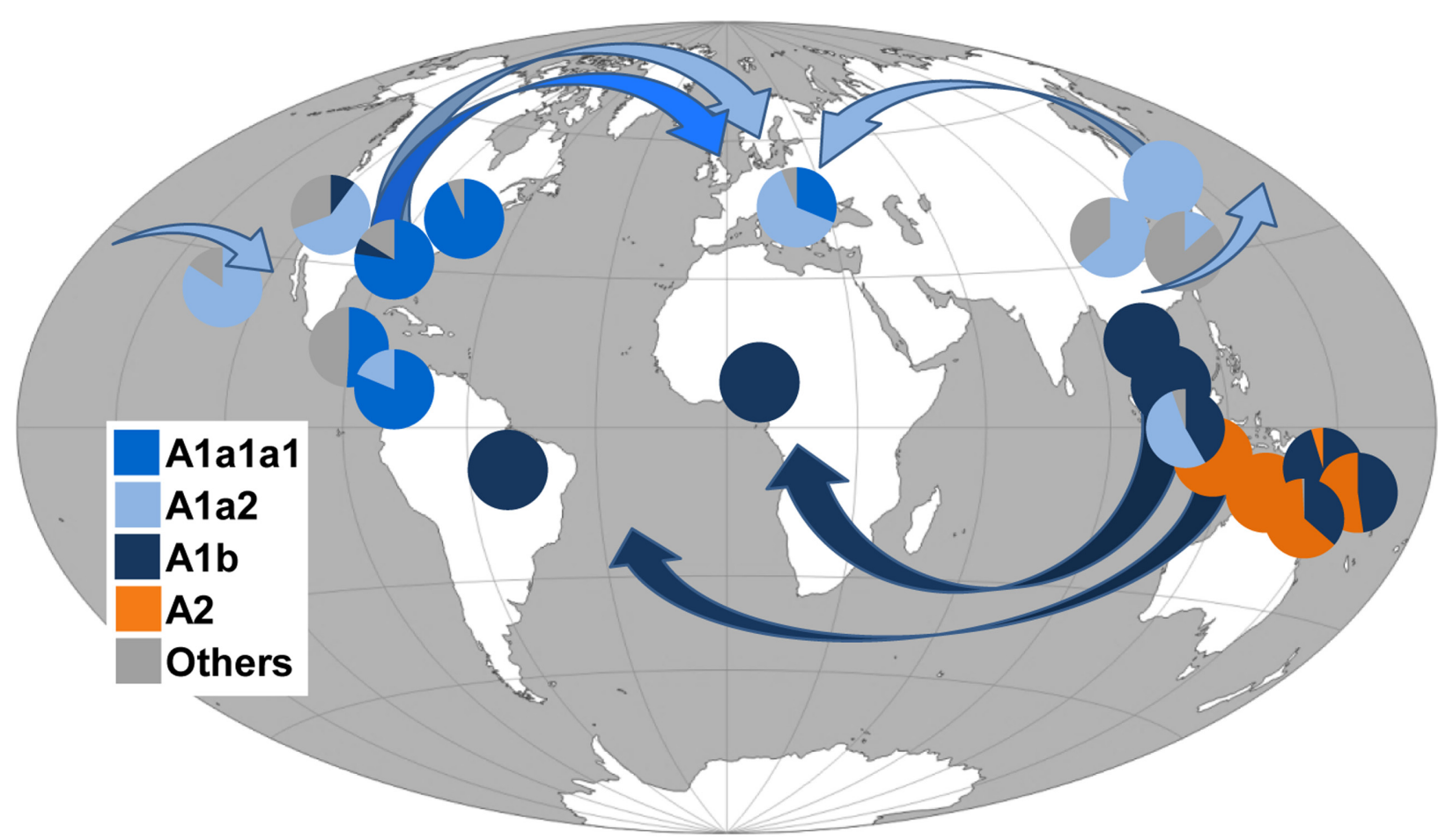

FIGURE 3 | Frequency (\%) of $\boldsymbol{A}$. albopictus mtDNA haplogroups in worldwide populations and possible diffusion routes. Arrows indicate possible diffusion routes from the native home-range (South-East Asia) and subsequent dispersals. Populations and corresponding frequency values are listed in Table 2. Note that the Chinese laboratory strain was not included. The original world map is from the website (http://www.freeworldmaps.net).

might be typical and possibly limited to the Philippines, Indonesia, Papua New Guinea, and Northern Australia (Table 2). Therefore, haplogroup A2 appears to have played a role in the spread of $A$. albopictus from South-East Asia (Figure 3) restricted to the context of Oceania. Indeed $A$. albopictus is thought to have spread from Indonesia by human-mediated transportation (Beebe et al., 2013). The low frequency of A2 observed on the Papua New Guinean mainland further supports this scenario, whereas the presence of both A1b and A2 mtDNAs along the North Australian border (Table 2) suggests multiple arrivals from distinct geographical sources (Beebe et al., 2013).

Figure 3 shows that, in contrast to the situation described above, members of the other three Asian haplogroups Ala1, $\mathrm{A} 1 \mathrm{a} 2$, and $\mathrm{A} 1 \mathrm{~b}$ are detected in many adventive populations worldwide. This finding identifies these as the Asian mtDNA lineages mainly associated with the recent global spread.

As for haplogroup Ala1, seen in the Japanese mitogenome \#26, it is widely distributed in Italy (Figure 1) and shows high frequencies in Central America and Eastern USA. Haplogroup A1a2 is present with frequencies higher than $50 \%$ in Japan, Southern China, Singapore, Hawaii, California and Italy, whereas A1b is fixed or almost fixed in Thailand, Malaysia, the Papua New Guinea mainland, Cameroon, and Brazil, but present at much lower frequencies also in California and Texas (Table 2). Even though these geographical distributions are based on the limited population sampling reported in Table 2, some preliminary conclusions can be drawn.

It appears that the ancestral homeland of haplogroup A1a2 might have been a temperate area, possibly Japan or Northern Asia, rather than the tropical range, in agreement with early allozyme studies (Kambhampati et al., 1991). In contrast haplogroup A1b appears to mainly characterize the tropical belt (Figure 3). This may imply that genetic and physiological traits make populations with A1b most suited to the colonization of tropical areas (Birungi and Munstermann, 2002; Kamgang et al., 2011). In our phylogeny the Brazilian A1b mitogenome (\#9) harbors a mutational motif that includes the transition at np 7210 in the ND5 gene, also found in all Brazilian samples retrieved from the literature and already identified as a marker for Brazilian A. albopictus (Birungi and Munstermann, 2002). The same transition was found in two ND5 haplotypes retrieved from the literature, one from Phuntsholing in Southern Bhutan (JQ436953) and one from Chiang Mai in Thailand (JQ436956; Porretta et al., 2012), suggesting a probable route of invasion from Indochina (Goubert et al., 2016). Instead the absence of this transition in the samples from Cameroon (Table 2) suggests that A1b mtDNAs arrived in Cameroon from a different tropical source. Interestingly, the absence of photoperiodic diapause in Brazilian mosquitoes supports their origin in tropical Asia, while the diapause in US populations is in agreement with the scenario of an ancestry in the Asian temperate regions (Mousson et al., 2005; Urbanski et al., 2010). 
As for haplogroup A1a1, its distribution could not be fully assessed because of the lack of informative marker mutations. However, the absence of the COI transitions at nps 1536 and 2165 distinguishes the members of its main sub-branch, Alala1 (Figure 1), from all other mitogenomes in the phylogeny. The survey of these transitions in published data sets revealed that haplogroup Alalal is the most common in Costa Rica, Panama, Texas, and New Jersey (Figure 3) and widespread in Italy (Table 2). This distribution suggests that Alalal and/or its derivatives Alalala and Alalalal arose recently in an adventive non-Asian population, probably from an ancestral Japanese source, and reached a high frequency because of genetic drift or founder events. From the earliest adventive non-Asian population(s) they then further spread to other distant regions. Such a possibility is in agreement with some previous observations, in particular with the suggestion that Italian tiger mosquitoes, whose first presence was documented in Northern Italy in 1990 (Sabatini et al., 1990), have a dual origin: a possibly direct Northern American source and a probably indirect (through Albania and Greece) Eastern Asian source, with the former related to the international trade of used tires from the eastern coast of the US (Dalla Pozza et al., 1994; Dritsou et al., 2015).

An origin of haplogroup Alalal and/or its derived subbranches Alalala and Alalalal in North America and a subsequent arrival to Italy from the US of multiple haplotypes is supported by (i) the detection of four partial sequences from Texas with the transition at np 1823 (Zhong et al., 2013), which defines sub-branch Alalalal, and (ii) our findings that mitogenomes \#4 and \#5, the first from Virginia and the second from Italy, are identical, and that mitogenomes \#2 and \#3, again one from Virginia and one from Italy, are closely related (Figure 1). Finally, the scenario that the ancestral source of Alalal might be a northern temperate area such as Japan is further supported by the presence of the COI transitions 1536 and 2435, which characterize mitogenome \#26, in five published mtDNAs from Kyoto (JQ004524; Xu and Fonseca, 2011), and is in agreement with allozyme data that have highlighted genetic links between North American, Italian and Japanese populations (Urbanelli et al., 2000).

\section{CONCLUSION}

Through our analyses, based on complete coding regions, the phylogeny of the A. albopictus mitogenomes was charted, and the most likely Asian sources of some adventive populations were identified. The worldwide spread of A. albopictus appears to be associated with three mtDNA haplogroups (A1a1, A1a2, and A1b), differently distributed in Asian populations living in temperate and tropical regions, whereas a fourth Asian haplogroup (A2) appears to be restricted to Insular SouthEast Asia. These ancestral genetic sources now coexist (and interbreed) in many of the recently colonized areas. This occurs not only in the field but also in the laboratory as attested by our detection of both A1a1a1 and A1a2al mitogenomes in the Rimini maintained strain, thus creating novel genomic combinations that might be one of the causes of the continuous and apparently growing capability of $A$. albopictus to expand its geographical range.

Note that fine scale mitogenome surveys, encompassing multiple specimens from a wide range of East Asian populations might prove to be an essential pre-requisite to controlling the spread of this mosquito and limiting its social, medical, and economic implications. With a precise identification of the source populations in Asia it will become possible to evaluate the extent and nature of their nuclear genome diversity and the possible selective advantages (e.g., production of cold or desiccation resistant eggs, zoophilic versus anthropophilic changes in feeding behavior) relative to other Asian A. albopictus populations that, by contrast, have not spread.

\section{AUTHOR CONTRIBUTIONS}

VB, PG, AT, and AO conceived and designed the experiments. $\mathrm{VB}, \mathrm{PG}$, and $\mathrm{SB}$ performed the experiments. VB, PG, MRC, AA, LMG, AT, and AO analyzed the data. PG, PAJ, X-GC, AA, OS, LMG, ARM, GG, AT, and AO contributed reagents/materials/analysis tools. VB, PG, GG, AT, and AO wrote the manuscript. All authors contributed to interpretation of data, reviewed and approved the manuscript.

\section{FUNDING}

This work was supported by the University of Pavia strategic theme "Toward a governance model for international migration: an interdisciplinary and diachronic perspective" (MIGRAT-ING), the EU-FP7 Research Infrastructures project (INFRAVEC) Grant 228421 (to GG), the National Natural Science Foundation of China (81420108024) (to X-GC), the Italian Ministry of Health (RF-2010-2318965) (to ARM), the Italian Ministry of Education, University and Research: Progetti Futuro in Ricerca 2012 (RBFR126B8I) (to AA and AO), and Progetti Ricerca Interesse Nazionale 2012 (2012JA4BTY) (to AA, AT, and OS). PG was supported by a fellowship from the Department of Biology and Biotechnology, University of Pavia.

\section{ACKNOWLEDGMENT}

The authors are grateful to Dr. Anca Macovei (Dipartimento di Biologia e Biotecnologie "L. Spallanzani," Pavia, Italy) and Dr. Kentaro Itokawa (Department of Medical Entomology, National Institute of Infectious Diseases, Tokyo, Japan), for their contributions to the collection of the A. albopictus samples from the Philippines and Japan, respectively.

\section{SUPPLEMENTARY MATERIAL}

The Supplementary Material for this article can be found online at: http://journal.frontiersin.org/article/10.3389/fgene.2016. 00208/full\#supplementary-material 


\section{REFERENCES}

Achilli, A., Olivieri, A., Pellecchia, M., Uboldi, C., Colli, L., Al-Zahery, N., et al. (2012). Mitochondrial genomes from modern horses reveal the major haplogroups that underwent domestication. Proc. Natl. Acad. Sci. U.S.A. 109, 2449-2454. doi: 10.1073/pnas.1111637109

Achilli, A., Olivieri, A., Soares, P., Lancioni, H., Hooshiar Kashani, B., Perego, U. A., et al. (2008). Mitochondrial genomes of extinct aurochs survive in domestic cattle. Curr. Biol. 18, R157-R158. doi: 10.1016/j.cub.2008.01.019

Armbruster, P., Damsky, W. E., Giordano, R., Birungi, J., Munstermann, L. E., and Conn, J. E. (2003). Infection of new- and old-world Aedes albopictus (Diptera: Culicidae) by the intracellular parasite Wolbachia: implications for host mitochondrial DNA evolution. J. Med. Entomol. 40, 356-360. doi: 10.1603/ 0022-2585-40.3.356

Beebe, N. W., Ambrose, L., Hill, L. A., Davis, J. B., Hapgood, G., Cooper, R. D., et al. (2013). Tracing the tiger: population genetics provides valuable insights into the Aedes (Stegomyia) albopictus invasion of the Australasian region. PLoS Negl. Trop. Dis. 7:e2361. doi: 10.1371/journal.pntd.0002361

Bellini, R., Calvitti, M., Medici, A., Carrieri, M., Celli, G., and Maini, S. (2007). "Use of the sterile insect technique against Aedes albopictus in Italy: first results of a pilot trial," in Area-Wide Control of Insect Pests, From Research to Field Implementation, eds M. J. B. Vreysen, A. S. Robinson, and J. Hendrichs (Dordrecht: Springer), 505-515.

Benedict, M. Q., Levine, R. S., Hawley, W. A., and Lounibos, L. P. (2007). Spread of the tiger: global risk of invasion by the mosquito Aedes albopictus. Vector Borne Zoonotic Dis. 7, 76-85. doi: 10.1089/vbz.2006.0562

Birungi, J., and Munstermann, L. E. (2002). Genetic structure of Aedes albopictus (Diptera: Culicidae) populations based on mitochondrial ND5 sequences: evidence for an independent invasion into Brazil and United States. Ann. Entomol. Soc. Am. 95, 125-132. doi: 10.1603/0013-8746(2002)095[0125: GSOAAD]2.0.CO;2

Bonizzoni, M., Gasperi, G., Chen, X., and James, A. A. (2013). The invasive mosquito species Aedes albopictus: current knowledge and future perspectives. Trends Parasitol. 29, 460-468. doi: 10.1016/j.pt.2013.07.003

Chen, X. G., Jiang, X., Gu, J., Xu, M., Wu, Y., Deng, Y., et al. (2015). Genome sequence of the Asian tiger mosquito, Aedes albopictus, reveals insights into its biology, genetics, and evolution. Proc. Natl. Acad. Sci. U.S.A. 112, E5907-E5915. doi: 10.1073/pnas.1516410112

Chenna, R., Sugawara, H., Koike, T., Lopez, R., Gibson, T. J., Higgins, D. G., et al. (2003). Multiple sequence alignment with the Clustal series of programs. Nucleic Acids Res. 31, 3497-3500. doi: 10.1093/nar/gkg500

Chouin-Carneiro, T., Vega-Rua, A., Vazeille, M., Yebakima, A., Girod, R., Goindin, D., et al. (2016). Differential susceptibilities of Aedes aegypti and Aedes albopictus from the Americas to Zika virus. PLoS Negl. Trop. Dis. 10:e0004543. doi: 10.1371/journal.pntd.0004543

Dalla Pozza, G. L., Romi, R., and Severini, C. (1994). Source and spread of Aedes albopictus in the Veneto region of Italy. J. Am. Mosq. Control Assoc. 10, 589-592.

Delatte, H., Bagny, L., Brengue, C., Bouetard, A., Paupy, C., and Fontenille, D. (2011). The invaders: phylogeography of dengue and chikungunya viruses Aedes vectors, on the South West islands of the Indian Ocean. Infect. Genet. Evol. 11, 1769-1781. doi: 10.1016/j.meegid.2011.07.016

Dritsou, V., Topalis, P., Windbichler, N., Simoni, A., Hall, A., Lawson, D., et al. (2015). A draft genome sequence of an invasive mosquito: an Italian Aedes albopictus. Pathog. Glob. Health 109, 207-220. doi: 10.1179/2047773215Y. 0000000031

Drummond, A. J., and Rambaut, A. (2007). BEAST: Bayesian evolutionary analysis by sampling trees. BMC Evol. Biol. 7:214. doi: 10.1186/1471-2148-7-214

Dueñas, J. C., Gardenal, C. N., Llinás, G. A., and Panzetta-Dutari, G. M. (2006). Structural organization of the mitochondrial DNA control region in Aedes aegypti. Genome 49, 931-937. doi: 10.1139/G06-053

Futami, K., Valderrama, A., Baldi, M., Minakawa, N., Marín Rodríguez, R., and Chaves, L. F. (2015). New and common haplotypes shape genetic diversity in Asian tiger mosquito populations from Costa Rica and Panamá. J. Econ. Entomol. 108, 761-768. doi: 10.1093/jee/tou028

Gasperi, G., Bellini, R., Malacrida, A. R., Crisanti, A., Dottori, M., and Aksoy, S. (2012). A new threat looming over the Mediterranean basin: emergence of viral diseases transmitted by Aedes albopictus mosquitoes. PLoS Negl. Trop. Dis. 6:e1836. doi: 10.1371/journal.pntd.0001836
Goubert, C., Minard, G., Vieira, C., and Boulesteix, M. (2016). Population genetics of the Asian tiger mosquito Aedes albopictus, an invasive vector of human diseases. Heredity (Edinb). 117, 125-134. doi: 10.1038/hdy.2016.35

Gubler, D. J. (2006). Dengue/dengue haemorrhagic fever: history and current status. Novartis Found. Symp. 277, 3-16,discussion16-22,71-13,251-253. doi: 10.1002/0470058005.ch2

Hebert, P. D., Cywinska, A., Ball, S. L., and deWaard, J. R. (2003). Biological identifications through DNA barcodes. Proc. Biol. Sci. 270, 313-321. doi: 10. 1098/rspb.2002.2218

Higa, Y., Toma, T., Tsuda, Y., and Miyagi, I. (2010). A multiplex PCR-based molecular identification of five morphologically related, medically important subgenus Stegomyia mosquitoes from the genus Aedes (Diptera: Culicidae) found in the Ryukyu Archipelago. Jpn. J. Infect. Dis. 63, 312-316.

Kambhampati, S., Black, W. C., and Rai, K. S. (1991). Geographic origin of the US and Brazilian Aedes albopictus inferred from allozyme analysis. Heredity (Edinb.) 67, 85-93. doi: 10.1038/hdy.1991.67

Kamgang, B., Brengues, C., Fontenille, D., Njiokou, F., Simard, F., and Paupy, C. (2011). Genetic structure of the tiger mosquito, Aedes albopictus, in Cameroon (Central Africa). PLoS ONE 6:e20257. doi: 10.1371/journal.pone.0020257

Kamgang, B., Ngoagouni, C., Manirakiza, A., Nakouné, E., Paupy, C., and Kazanji, M. (2013). Temporal patterns of abundance of Aedes aegypti and Aedes albopictus (Diptera: Culicidae) and mitochondrial DNA analysis of Ae. albopictus in the Central African Republic. PLoS Negl. Trop. Dis. 7:e2590. doi: 10.1371/journal.pntd.0002590

Khormi, H. M., and Kumar, L. (2014). Climate change and the potential global distribution of Aedes aegypti: spatial modelling using GIS and CLIMEX. Geospat. Health 8, 405-415. doi: 10.4081/gh.2014.29

Kraemer, M. U., Sinka, M. E., Duda, K. A., Mylne, A., Shearer, F. M., Brady, O. J., et al. (2015). The global compendium of Aedes aegypti and Ae. albopictus occurrence. Sci. Data 2:150035. doi: 10.1038/sdata.2015.35

Kumar, S., Stecher, G., and Tamura, K. (2016). MEGA7: molecular evolutionary genetics analysis version 7.0 for bigger datasets. Mol. Biol. Evol. 33, 1870-1874. doi: 10.1093/molbev/msw054

Manni, M., Gomulski, L. M., Aketarawong, N., Tait, G., Scolari, F., Somboon, P., et al. (2015). Molecular markers for analyses of intraspecific genetic diversity in the Asian tiger mosquito, Aedes albopictus. Parasit. Vectors 8:188. doi: 10.1186/ s13071-015-0794-5

Medlock, J. M., Hansford, K. M., Schaffner, F., Versteirt, V., Hendrickx, G. Zeller, H., et al. (2012). A review of the invasive mosquitoes in Europe: ecology, public health risks, and control options. Vector Borne Zoonotic Dis. 12, 435-447. doi: 10.1089/vbz.2011.0814

Medlock, J. M., Hansford, K. M., Versteirt, V., Cull, B., Kampen, H., Fontenille, D., et al. (2015). An entomological review of invasive mosquitoes in Europe. Bull. Entomol. Res. 105, 637-663. doi: 10.1017/S000748531 5000103

Mousson, L., Dauga, C., Garrigues, T., Schaffner, F., Vazeille, M., and Failloux, A. B. (2005). Phylogeography of Aedes (Stegomyia) aegypti (L.) and Aedes (Stegomyia) albopictus (Skuse) (Diptera: Culicidae) based on mitochondrial DNA variations. Genet. Res. 86, 1-11. doi: 10.1017/S0016672305007627

Nei, M., and Kumar, S. (2000). Molecular Evolution and Phylogenetics. New York, NY: Oxford University Press.

Paupy, C., Delatte, H., Bagny, L., Corbel, V., and Fontenille, D. (2009). Aedes albopictus, an arbovirus vector: from the darkness to the light. Microbes Infect. 11, 1177-1185. doi: 10.1016/j.micinf.2009.05.005

Peng, H. J., Lai, H. B., Zhang, Q. L., Xu, B. Y., Zhang, H., Liu, W. H., et al. (2012). A local outbreak of dengue caused by an imported case in Dongguan China. BMC Public Health 12:83. doi: 10.1186/1471-2458-12-83

Porretta, D., Mastrantonio, V., Bellini, R., Somboon, P., and Urbanelli, S. (2012). Glacial history of a modern invader: phylogeography and species distribution modelling of the Asian tiger mosquito Aedes albopictus. PLoS ONE 7:e44515. doi: 10.1371/journal.pone.0044515

Powell, J. R., and Tabachnick, W. J. (2013). History of domestication and spread of Aedes aegypti-a review. Mem. Inst. Oswaldo Cruz 108, 11-17. doi: 10.1590/ 0074-0276130395

Rai, K. S. (1991). Aedes albopictus in the Americas. Annu. Rev. Entomol. 36, 459-484. doi: 10.1146/annurev.en.36.010191.002331

Rezza, G. (2012). Aedes albopictus and the reemergence of dengue. BMC Public Health 12:72. doi: 10.1186/1471-2458-12-72 
Rezza, G. (2014). Dengue and chikungunya: long-distance spread and outbreaks in naïve areas. Pathog. Glob. Health 108, 349-355. doi: 10.1179/2047773214Y. 0000000163

Romi, R., Sabatinelli, G., Savelli, L. G., Raris, M., Zago, M., and Malatesta, R. (1997). Identification of a north American mosquito species, Aedes atropalpus (Diptera: Culicidae), in Italy. J. Am. Mosq. Control. Assoc. 13, 245-246.

Rueda, L. M. (2004). Pictorial keys for the identification of mosquitoes (Diptera: Culicidae) associated with dengue virus transmission. Zootaxa 589, 1-60. doi: 10.11646/zootaxa.589.1.1

Sabatini, A., Raineri, V., Trovato, G., and Coluzzi, M. (1990). Aedes albopictus in Italy and possible diffusion of the species into the Mediterranean area. Parassitologia 32, 301-304.

Schaffner, F., Medlock, J. M., and Van Bortel, W. (2013). Public health significance of invasive mosquitoes in Europe. Clin. Microbiol. Infect. 19, 685-692. doi: 10.1111/1469-0691.12189

Scholte, E. J., Den Hartogm, W., Braks, M., Reusken, C., Dik, M., and Hessels, A. (2009). First report of a north American invasive mosquito species Ochlerotatus atropalpus (Coquillett) in the Netherlands, 2009. Euro Surveill. 14:19400.

Sumruayphol, S., Apiwathnasorn, C., Ruangsittichai, J., Sriwichai, P., Attrapadung, S., Samung, Y., et al. (2016). DNA barcoding and wing morphometrics to distinguish three Aedes vectors in Thailand. Acta Trop. 159, 1-10. doi: 10.1016/j.actatropica.2016.03.010

Torroni, A., Achilli, A., Macaulay, V., Richards, M., and Bandelt, H. J. (2006). Harvesting the fruit of the human mtDNA tree. Trends Genet. 22, 339-345. doi: 10.1016/j.tig.2006.04.001

Urbanelli, S., Bellini, R., Carrieri, M., Sallicandro, P., and Celli, G. (2000). Population structure of Aedes albopictus (Skuse): the mosquito which is colonizing Mediterranean countries. Heredity (Edinb) 84, 331-337. doi: 10. 1046/j.1365-2540.2000.00676.x

Urbanski, J. M., Benoit, J. B., Michaud, M. R., Denlinger, D. L., and Armbruster, P. (2010). The molecular physiology of increased egg desiccation resistance during diapause in the invasive mosquito, Aedes albopictus. Proc. Biol. Sci. 277, 2683-2692. doi: 10.1098/rspb.2010.0362

Wong, P. S., Li, M. Z., Chong, C. S., Ng, L. C., and Tan, C. H. (2013). Aedes (Stegomyia) albopictus (Skuse): a potential vector of Zika virus in Singapore. PLoS Negl. Trop. Dis. 7:e2348. doi: 10.1371/journal.pntd.0002348
Wu, J. Y., Lun, Z. R., James, A. A., and Chen, X. G. (2010). Dengue fever in mainland China. Am. J. Trop. Med. Hyg. 83, 664-671. doi: 10.4269/ajtmh.2010. 09-0755

Xu, J., and Fonseca, D. M. (2011). One-way sequencing of multiple amplicons from tandem repetitive mitochondrial DNA control region. Mitochondrial DNA 22, 155-158. doi: 10.3109/19401736.2011.636434

Yang, Z. (2007). PAML 4: phylogenetic analysis by maximum likelihood. Mol. Biol. Evol. 24, 1586-1591. doi: 10.1093/molbev/msm088

Zawani, M. K., Abu, H. A., Sazaly, A. B., Zary, S. Y., and Darlina, M. N. (2014). Population genetic structure of Aedes albopictus in Penang, Malaysia. Genet. Mol. Res. 13, 8184-8196. doi: 10.4238/2014.October.7.13

Zhang, D.-X., and Hewitt, G. M. (1997). Insect mitochondrial control region: a review of its structure, evolution and usefulness in evolutionary studies. Biochem. Syst. Ecol. 25, 99-120. doi: 10.1016/S0305-1978(96) 00042-7

Zhang, H., Xing, D., Wang, G., Li, C., and Zhao, T. (2015). Sequencing and analysis of the complete mitochondrial genome of Aedes albopictus (Diptera: Culicidae) in China. Mitochondrial DNA A DNA Mapp Seq Anal. 27, 2787-2788. doi: 10.3109/19401736.2015.1053067

Zhong, D., Lo, E., Hu, R., Metzger, M. E., Cummings, R., Bonizzoni, M., et al. (2013). Genetic analysis of invasive Aedes albopictus populations in Los Angeles County, California and its potential public health impact. PLoS ONE 8:e68586. doi: 10.1371/journal.pone. 0068586

Conflict of Interest Statement: The authors declare that the research was conducted in the absence of any commercial or financial relationships that could be construed as a potential conflict of interest.

Copyright (c) 2016 Battaglia, Gabrieli, Brandini, Capodiferro, Javier, Chen, Achilli, Semino, Gomulski, Malacrida, Gasperi, Torroni and Olivieri. This is an open-access article distributed under the terms of the Creative Commons Attribution License (CC BY). The use, distribution or reproduction in other forums is permitted, provided the original author(s) or licensor are credited and that the original publication in this journal is cited, in accordance with accepted academic practice. No use, distribution or reproduction is permitted which does not comply with these terms. 